\title{
A single-domain antibody inhibits SFTSV and mitigates virus-induced pathogenesis in vivo
}

\author{
Xilin Wu, ${ }^{1}$ Yanlei Li, ${ }^{2}$ Bilian Huang, ${ }^{1}$ Xiaohua Ma, ${ }^{3}$ Linjing Zhu, ${ }^{4}$ Nan Zheng, ${ }^{1}$ Shijie Xu, ${ }^{1}$ \\ Waqas Nawaz, ${ }^{1}$ Changping $\mathrm{Xu},{ }^{1}$ and $\mathrm{Zhiwei} \mathrm{Wu}^{3,5,6}$
}

${ }^{1}$ Center for Public Health Research, Medical School, Nanjing University, Nanjing, China. ${ }^{2} Y$-Clone Medical Science Co. Ltd., Suzhou, China. ${ }^{3}$ School of Life Sciences, Ningxia University, Yinchuan, China. ${ }^{4}$ Abrev Biotechnology Co., Ltd., Nanjing, China. ${ }^{5}$ Jiangsu Key Laboratory of Molecular Medicine, Medical School, Nanjing University, Nanjing, China. ${ }^{6}$ State Key Laboratory of Analytical Chemistry for Life Science, Nanjing University, Nanjing, China.

Severe fever with thrombocytopenia syndrome virus (SFTSV) is a novel tick-borne bunyavirus that recently emerged in East Asian countries. SFTS is characterized by high fever, thrombocytopenia, leukopenia, multiorgan failure, and hemorrhage with case fatality rates of $6.3 \%$ to $30 \%$. Neither antivirals nor vaccines are available at present. We previously demonstrated that neutralizing antibodies specific for SFTSV glycoprotein (Gn) played a vital role in the survival of patients with SFTS. Nanobodies from camels present unique properties, such as thermostability, high affinity, and low immunogenicity. In the current study, mammalian expressed SFTSV Gn was used to immunize a camel, and functional nanobodies were isolated from the B cell nanobody library constructed from the immunized animal. Clone SNBO2 was selected for in-depth analysis for its inhibition of SFTSV replication both in vitro and in vivo. We showed that SNBO2 potently inhibited SFTSV infection and prevented thrombocytopenia in a humanized mouse model and is a potential candidate for therapeutics.

Authorship note: XW and YL contributed equally to this work.

Conflict of interest: A patent application for SNBO2 was submitted by Y-Clone Medical Science Co. Ltd., under PCT/CN2019/097350. YL is listed on the application.

Copyright: (ㄷ) 2020, American Society for Clinical Investigation.

Submitted: February 6, 2020

Accepted: June 3, 2020

Published: July 9, 2020.

Reference information: /CI Insight. 2020;5(13):e136855.

https://doi.org/10.1172/jci.

insight.136855.

\section{Introduction}

Severe fever with thrombocytopenia syndrome (SFTS) is an acute infectious disease caused by a novel bunyavirus (severe fever with thrombocytopenia syndrome virus, SFTSV). The major clinical findings are characterized by high fever, thrombocytopenia, leukopenia, and multiple organ dysfunctions, including the lung, gastrointestinal tract, liver, and kidney (1-3), with high mortality rates of $6.3 \%$ to $30 \%$ (4). Ticks such as Haemaphysalis longicornis and Rhipicephalus microplus are implicated as the prominent vectors for transmission while human-to-human transmission through contact with blood or body fluids of patients with SFTS was reported $(5,6)$. Furthermore, recently this infection was also reported in pets (7-9). Since the first SFTS case was reported in China in 2009, the incidence of SFTS has expanded to at least 15 provinces in China (10) and has been reported in South Korea, in Japan, and recently in Vietnam, suggesting that the endemic area is expanding (10). To date, no specific treatment for SFTS is available.

Studies have shown that neutralizing antibodies against SFTSV surface glycoprotein (glycoprotein N, $\mathrm{Gn}$ ) are highly associated with patient survival (11-13). Our previous study in a patient cohort demonstrated that the presence of serum anti-Gn antibodies was correlated with survival, while the mortality of SFTS patients without serum anti-Gn antibodies was as high as $66.7 \%(14)$. These studies suggest that neutralizing antibodies specific for $\mathrm{G}$ play protective roles in the infection and $\mathrm{Gn}$ is a potential target for vaccine or therapeutic antibody development.

Variable heavy chain domain $(\mathrm{VHH})$ is an immunoglobulin single variable domain $(12-15 \mathrm{kDa})$ of heavy chain antibodies that occurs naturally in camelids (15). VHH is the smallest functional antibody fragment currently known, which is also called nanobody or single-domain antibody $(15,16)$. Recently, nanobody has been widely studied for various applications due to its high solubility, thermostability, low toxicity, and low immunogenicity. Nanobody drugs for tumors, inflammation, infectious diseases, and neurological diseases are under development $(17,18)$. Caplacizumab was approved by the FDA in 2019 as the first camel-derived 
monoclonal antibody drug for acquired thrombotic thrombocytopenic purpura (19). Other nanobody drugs, such as KN035 for tumors and ALX-0061 for inflammation, are under clinical trials $(18,20)$.

In the present study, the extracellular domain of SFTSV Gn (sGn) expressed in mammalian cells was used to immunize a camel, and peripheral blood mononuclear cells (PBMCs) were isolated from the immunized camel for a VHH antibody phage library construction. After multiple rounds of enrichment against sGn, 23 nanobodies with potent neutralizing activities were identified. SNB02, one of the nanobodies fused with a human $\mathrm{CH} 2-3$ (VHH-huFc, named SNB), potently inhibited SFTSV infection and prevented thrombocytopenia in a humanized mouse model and is a potential therapeutic agent for SFTS.

\section{Results}

Induction and characterization of antisera. A camel was immunized with sGn 4 times with an immunization regimen as shown in Figure $1 \mathrm{~A}$. sGns with His or rabbit $\mathrm{Fc}(\mathrm{rFc})$ tag were expressed, purified, and characterized by SDS-PAGE (Figure 1B). The antiserum titer reached $4.61 \times 10^{6}$ after the fourth immunization (Figure 1C). Immunofluorescence assay showed that the fourth antisera specifically recognized the 293TT cells transfected with Gn plasmids of various SFTSV subtypes, including subtypes A-E, indicating that the fourth camel antisera could broadly react with different subtypes of SFTSV (Figure 1D). The camel antiserum was shown to inhibit SFTSV infection with an $\mathrm{ID}_{90}$ at 540 -fold dilution in a preliminary screening (Supplemental Figure 1; supplemental material available online with this article; https://doi.org/10.1172/ jci.insight.136855DS1). In summary, sGn induced high titer antisera that were broadly reactive with various subtypes of SFTSV with potent neutralization.

Identification of $s \mathrm{Gn}$-VHH phage library. A phage library displaying $\mathrm{VHH}$ antibody was constructed to isolate sGn-specific VHH antibodies by isolating PBMCs from the immunized camel, extracting RNA to reverse-transcribe into cDNA, and amplifying the VHH gene (Supplemental Figure 2, A-C). The sGnVHH phage library was validated by PCR amplification of the VHH target gene of $400 \mathrm{bp}$ with expected size (Supplemental Figure 2D), suggesting that the sGn-VHH phage library contains VHH. To further characterize the sGn-VHH phage library, 50 single bacterial clones were selected for sequencing, and the results showed that the sequences were in frame to encode VHH antibody and were not completely identical (Supplemental Figure 3). The alignment showed that the sequence differences were mostly in the complementarity-determining regions (CDRs). In summary, an sGn-VHH phage antibody library was constructed with a size of $2.0 \times 10^{9}, 100 \%$ sequence diversity, and $98 \%$ in-frame rate (Table 1 ).

In order to isolate $\mathrm{VHH}$ antibodies specific for $\mathrm{Gn}, 3$ rounds of biopanning on the sGn-VHH phage library were conducted. The panned library was analyzed by phage ELISA to test the binding with sGn, and the results showed that the $\mathrm{OD}_{450}$ readout increased from 0.57 before enrichment to $0.98,2.2$, and 3.0 after the first, second, and third rounds of enrichment, respectively (Figure 2A), suggesting successful enrichment. In order to verify whether the enriched library contained specific sGn-reactive phages, 96 clones were selected from the libraries after the second and third rounds of enrichment for single-phage ELISA. The percentage of positive clones was $24.5 \%$ and $67 \%$ for the second and third rounds, respective$1 \mathrm{y}$, and the average $\mathrm{OD}_{450}$ readout was around 3.0, suggesting high-affinity VHH binding with sGn by the sGn-VHH phage library (Figure 2, A and B; and Supplemental Table 1).

High-throughput evaluation of VHH function. We developed a high-throughput assay for early evaluation of antibody function by expressing VHHs in SS320 bacteria. The bacterial VHH antibody library was constructed based on the plasmids of the second and third round phages. A total of 1399 colonies were randomly selected for the evaluation of the binding with $\mathrm{sGn}$ in an ELISA format, and 197 bacterial clones with sGn/blank binding ratio greater than 5.0 were selected (Figure 2C and Supplemental Table 2). All 197 clones were sequenced and aligned, and 142 distinct VHH sequences were obtained (Supplemental Figure 4A and Supplemental Table 3). Sequence analysis of the isolated 142 distinct VHHs revealed that VHH sequences and repertoires of CDRs were highly diverse, with CDR1, CDR2, and CDR3 each containing 65, 69, and 72 unique lineages, respectively (Supplemental Figure 4, A and B). The distribution of CDR lengths in sGn-specific nanobody repertoires was skewed toward lengths of 8 amino acids for CDR1 and CDR2 and of 20 amino acids for CDR3 (Supplemental Figure 4C). The 197 clones were analyzed for their neutralization activities against SFTSV infection, and some exhibited potent inhibitory activities with more than $90 \%$ inhibition. Twenty-three $\mathrm{VHH}$ antibodies that reached $\mathrm{IC}_{50}$ were selected for further evaluation (Figure 2D). In summary, 23 unique VHH antibodies specific for sGn with potent neutralization activity against SFTSV infection were obtained. 
A

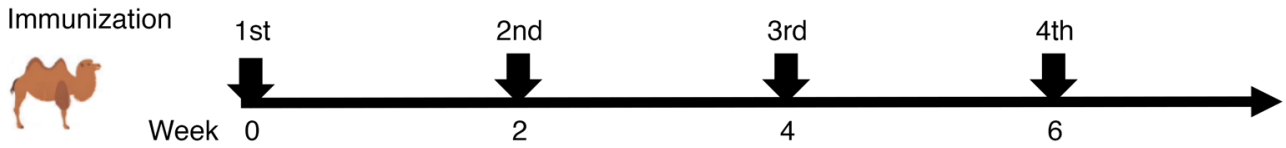

B

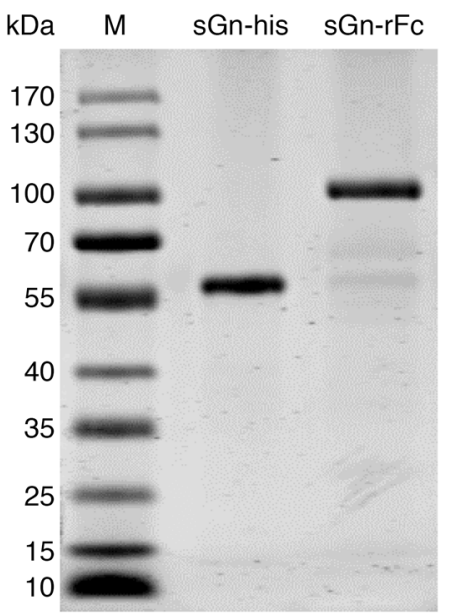

C

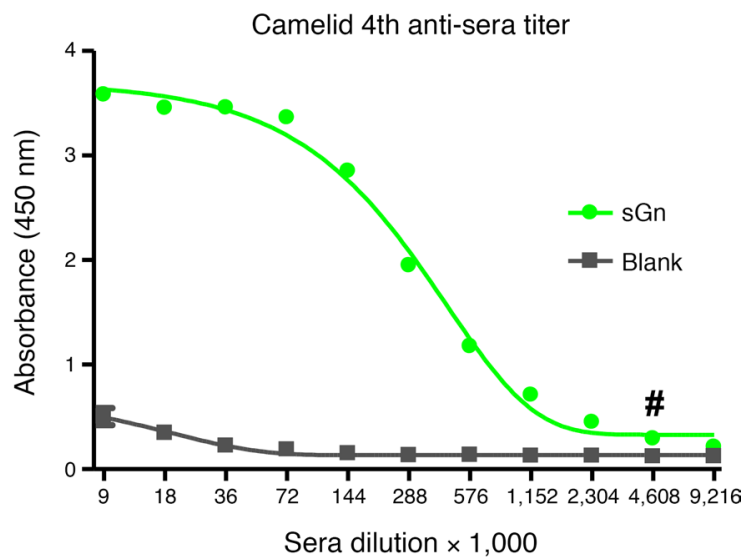

D SFTSV Gn subtype
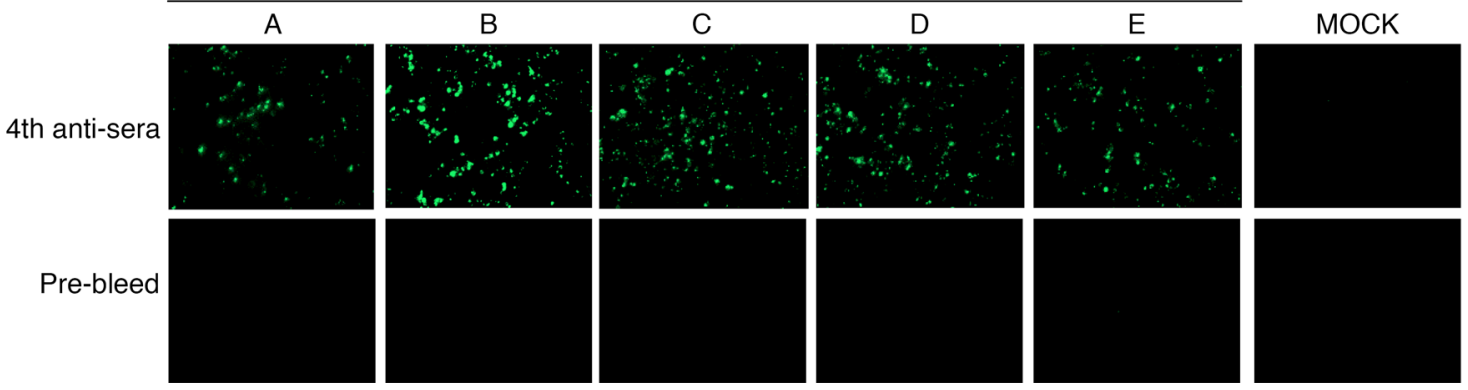

Figure 1. Characterization of antisera specific for sGn. (A) The experimental schedule of immunization; (B) sGn protein with his tag (sGn-his) or rabbit Fc tag (sGn-rFc) were detected by SDS-PAGE. (C) The titer of antisera was evaluated after the fourth immunization in camel receiving sGn. The $y$ axis represents the absorbance at $450 \mathrm{~nm} ; x$ axis, the antisera dilution fold. Antisera binding with $\mathrm{sGn}$ and coating buffer were labeled as sGn with green line and labeled as blank with black line, respectively. \# represents the antiserum titer. (D) Determination of the specificity of antisera by immunofluorescence assay. 293TT cells were transiently transfected with Gn plasmid of SFTSV subtypes A, B, C, D, and E, and the cells were stained with the camel fourth antisera and sera preimmunization (Pre-bleed) for $\mathrm{Gn}$ (green). Mock served as a cell control without plasmid transfection. Images were visualized under the $\times 20$ objective. All experiments of $\mathbf{B}-\mathbf{D}$ were repeated twice.

Construction and characterization of VHH-human Fc1. VHH antibodies were expressed in mammalian cells by fusing VHH gene with an huFc, which was cloned into the pVAX1 vector to express VHH-huFc antibody (SNB) (Figure 3A). The purified SNBs from transfected 293-F cell supernatants were tested to neutralize live SFTSV infection in Vero E6 cells. SNB02 exhibited one of the most potent neutralization activities, with an $\mathrm{IC}_{50}$ of $1.05 \mu \mathrm{g} / \mathrm{mL}$ (Figure 3B). SNB binding to sGn was analyzed by both ELISA and surface plasmon resonance (SPR), which showed that SNB02 presented one of the best binding activities with the fastest on rate with the highest association curve (Figure 3, C and D). Most SNBs exhibited high affinities in the order of picomolar (Supplemental Figure 5). SNB02, representing one of the best SNBs, possessed potent in vitro neutralization activity against SFTSV and exhibited a very fast binding and slow dissociation (Figure 3D).

SNB02 inhibits SFTSV and prevents thrombocytopenia in a humanized mouse model. Given that SNB02 exhibited high affinity and one of the most potent neutralizing activities among the SNBs, SNB02 was chosen for further functional analysis both in vitro and in vivo. The purified SNBs from transfected 293-F 
Table 1. The summary of the sGn-VHH library

\begin{tabular}{ccccccc}
\hline Name & Library size & Sequencing clone & Sequence results & In-frame clone & Diversity & In-frame rate \\
SGN-VHH-lib & $2 \times 10^{9}$ & 50 & 45 & 44 & $44 / 44(100 \%)$ & $44 / 45(98 \%)$ \\
\hline
\end{tabular}

cell supernatants were analyzed by SDS-PAGE (Figure 4A), and SNB02's purity was determined by size exclusion chromatography to be $97.88 \%$ (Figure 4B). SNB02 reacted with both reduced and nonreduced sGn protein as determined by Western blot (Figure 4C), suggesting that SNB02 likely recognizes a conformational epitope on sGn. SNB02 exhibited binding activities with sGn of subtypes A-E based on ELISA and Western blot results, indicating SNB02 cross-reactivity with various SFTSV subtypes (Supplemental Figure 6). The kinetic binding of SNB02 with sGn protein is $5.479 \times 10^{9} \mathrm{M}$ (Figure 4D and Supplemental Figure 5A). SNB02 inhibited SFTSV infection of PBMCs and Vero E6 cells with an $\mathrm{IC}_{50}$ at less than 2.7 $\mu \mathrm{g} / \mathrm{mL}$, indicating that SNB02 potently inhibited not only SFTSV infection of the Vero E6 cell line but also SFTSV infection of PBMCs (Figure 4E). The half-life of SNB02 was 9.5 hours in the NOD.Cg-Prkdcsid Il2r$g^{e m 26} / \mathrm{Nju} \mathrm{HuPBL}$ (NCG-HuPBL) mouse model (Figure 4F). In summary, SNB02 was produced with high purity, recognizing a conformational epitope of sGn with high affinity. SNB02 potently inhibited SFTSV infection in PBMCs and Vero E6 cells.

Encouraged by the potent in vitro antiviral activity, we next evaluated the SNB02 against SFTSV in a humanized NCG mouse model. SFTSV-infected, humanized mice $(n=8)$ were treated with 400 $\mu \mathrm{g}$ /mouse SNB02 1 hour, 1 day, 2 days, and 3 days postinfection or 1 day, 2 days, 3 days, and 4 days postinfection (Figure $5 \mathrm{~A}$ ). Infected mice treated with PBS at the same time intervals served as controls. Compared with the control mice, SFTSV replication was significantly inhibited by the SNB02 treatment (Figure 5B), with viremia reduced more than $2 \log _{10}$ for the group with SNB02 administrated at 1 hour postinfection and $1 \log _{10}$ for the group with SNB02 administrated at 24 hours postinfection. Interestingly, platelet counts in the SNB02-treated mice were significantly higher than in the control group, suggesting that SNB02 prevented thrombocytopenia (Figure 5C). The correlational analysis shows that the viral load was inversely correlated with the platelet counts (Figure 5D). The SNB02-treated mice showed no significant weight loss or leukocytosis (WBC count, neutrophils, lymphocytes, and monocytes) as compared with the control (Figure 6). In summary, SNB02 not only inhibited SFTSV replication but also prevented thrombocytopenia in a humanized mouse model infected by SFTSV.

SFTSV infection causes vascular permeability, resulting in hemorrhagic outcomes in the infected patients, a hallmark of the SFTS pathogenesis and one of the major risk factors for death (2). Thus we investigated the effect of SNB02 treatment on vascular permeability. Vascular endothelial hyperpermeability was detected in the lung 8 days after SFTSV infection of mice compared with noninfected mice (Figure 5E). Interestingly, SNB02 treatment prevented the vascular endothelial hyperpermeability caused by SFTSV infection (Figure 5E). SFTS is also characterized by respiratory tract symptoms and multiple organ failure, including lung, liver, and kidney, followed by a progressive decline in platelets. H\&E-stained tissues of the above mice showed that SNB02 could ameliorate the infiltration of inflammatory cells in the lung, liver, and glomerular hyperemia in the kidney caused by SFTSV infection (Figure 7). Taken together, the results indicate that SNB02 could not only inhibit viral replication but also ameliorate virus-induced pathogenesis.

\section{Discussion}

SFTSV infection and related death have been on the rise with increasing incidence and an expanding endemic in East Asian countries with clinical fatality rates of $6 \%$ to $30 \%$ (4). Ticks are implicated as the prominent vectors for transmitting SFTSV, and various vertebrate species are considered susceptible to the infection, as evidenced by high SFTSV seroprevalence in animals like cats, dogs, birds, water deer, and wild boar in SFTS-endemic regions, suggesting that SFTSV infection widely exists (21). Human-to-human transmission through contact with blood or body fluids of patients with SFTS was reported $(6,22)$, posing a further threat to the public health. Spatial distributions of SFTSV cases are mainly reported in Asian countries, such as China, South Korea, Japan, and Vietnam, and $0.8 \%$ to $7.2 \%$ people in counties of endemic region are considered to have been infected, as evidenced by high SFTSV seroprevalence (23). SFTSV transmission 
A

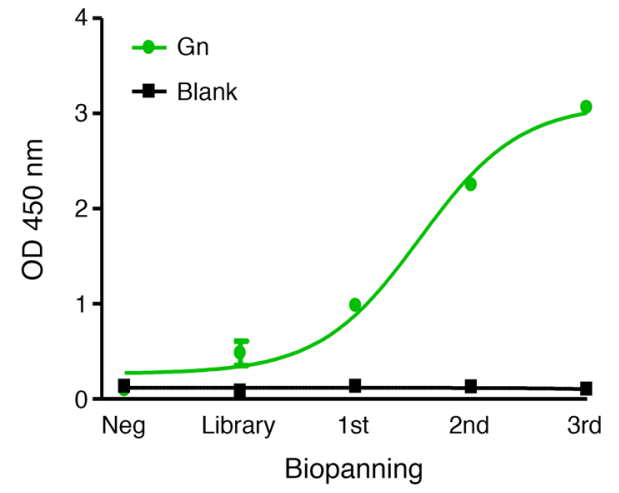

C

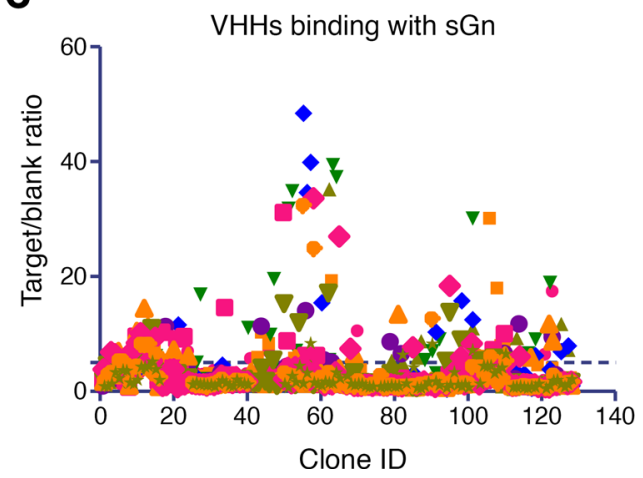

B

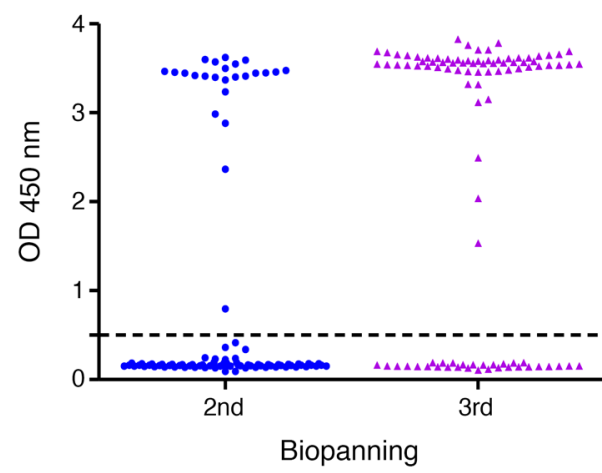

D

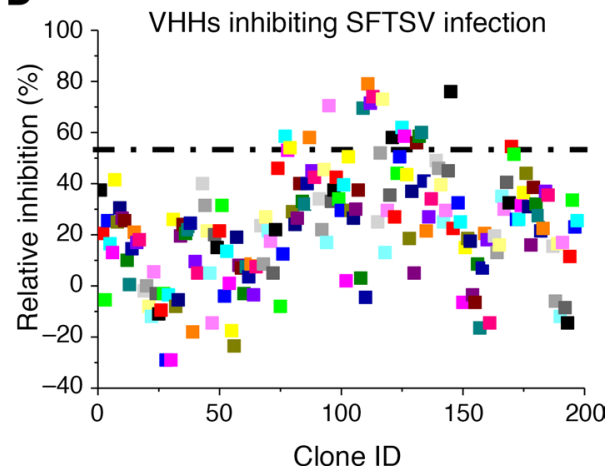

Figure 2. The biopanning and screening of the sGn-VHH antibody phage library. (A) The binding of the phage library with sGn via phage ELISA. Lib is the phage library of $s \mathrm{Gn}$ VHH; 1st, 2nd, and 3rd are the phage library after panning on 1 round, 2 rounds, and 3 rounds of sGn enrichment, respectively. (B) Ninety-five single clones of phages from the sGn-VHH library after the second and third enrichment of sGN were analyzed by phage ELISA. (C) The summary of bacterial supernatant binding with SGn protein tested by phage ELISA. The $y$ axis shows the ratio of the readout of sGn binding to the readout of blank binding at OD $450 \mathrm{~nm}$. Each square represents bacterial supernatant from a single clone of 1399 clones. (D) Positive binding VHHs inhibiting SFTSV infection, where each dot represents $1 \mathrm{VHH}$ clone supernatant. Dashed line is located at 50\% relative inhibition.

by ticks in domestic and migratory birds increases the potential spatial distribution of SFTSV outside Asia. Haemaphysalis longicornis, the major host of SFTSV in Asia, was positively confirmed in the United States in September 2018, indicating that spatial distribution of SFTSV may have already been outside Asia (24). Therefore, there is an urgent need for therapeutics or vaccines for SFTSV infection in humans and animals.

Two anti-Gn SFTS neutralizing antibodies, Mab4-5 and Ab10, isolated from a phage display library of SFTS patient antibodies, were reported earlier $(25,26)$. Only Ab10 was evaluated for the inhibition of SFTSV infection in an A129 mouse model without monitoring SFTSV titer in vivo during the treatment (26). In the current study, we reported the isolation of Gn-specific single-domain antibodies from an immunized camel and the comprehensive characterization of the neutralizing activity of the nanobody.

SFTSV infection has been studied in various animal models, including mice, hamsters, and ferrets, with various successes. SFTSV-infected C57/BL6 mice were reported to present symptoms of thrombocytopenia and leukocytopenia. However, viral replication could not be observed in blood. Even in the spleen, viral replication could only be observed 3 days postinfection (27). In another murine model, $\alpha / \beta$ interferon receptor-knockout $\left(\mathrm{IFNAR}^{--}\right)$mice, the course of SFTSV infection is short, and the infected mice die within 3 to 4 days with high viral load, which makes the pathogenesis distinct from the human infection and allows only a very narrow window for testing drugs. In addition, SFTSV infected reticular cells in the IFNAR $^{-/-}$mice instead of monocytes/macrophages in human. No thrombocytopenia with a progressive decline in platelets and white blood cells was reported in the SFTSV-infected IFNAR ${ }^{-/}$mice (28).

SFTSV-infected aged ferrets (4 years of age) demonstrated severe thrombocytopenia, reduced WBC counts, and high fever with high mortality rate. However, ferrets are outbred animals unlike inbred mice, and of note, aged ferrets are quite limited (29). In addition, the efficacy of humanized antibody was 
A

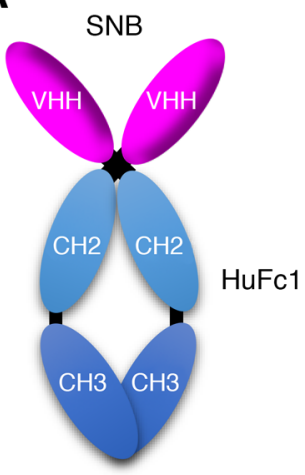

B

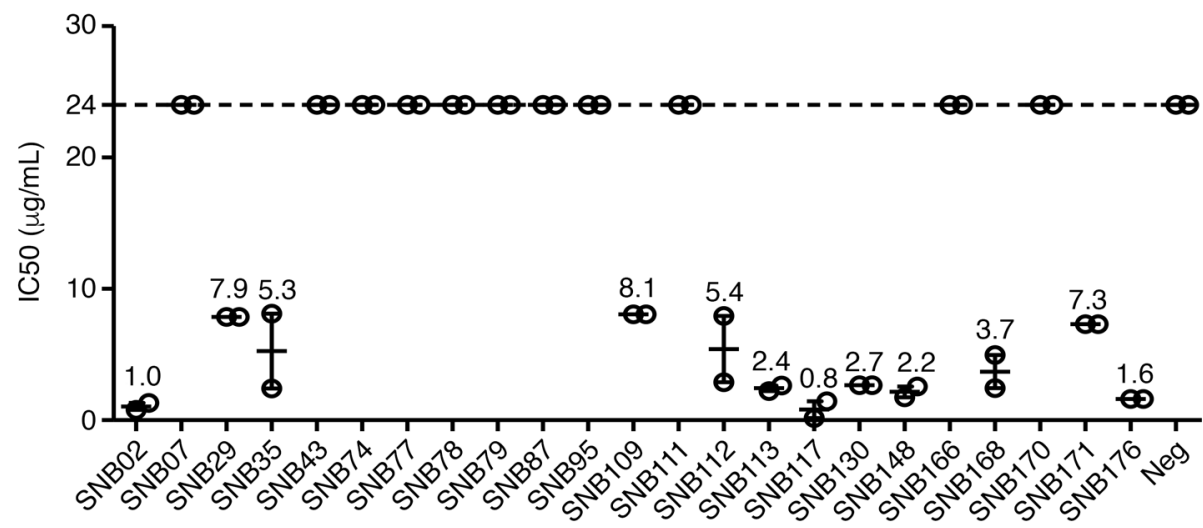

C
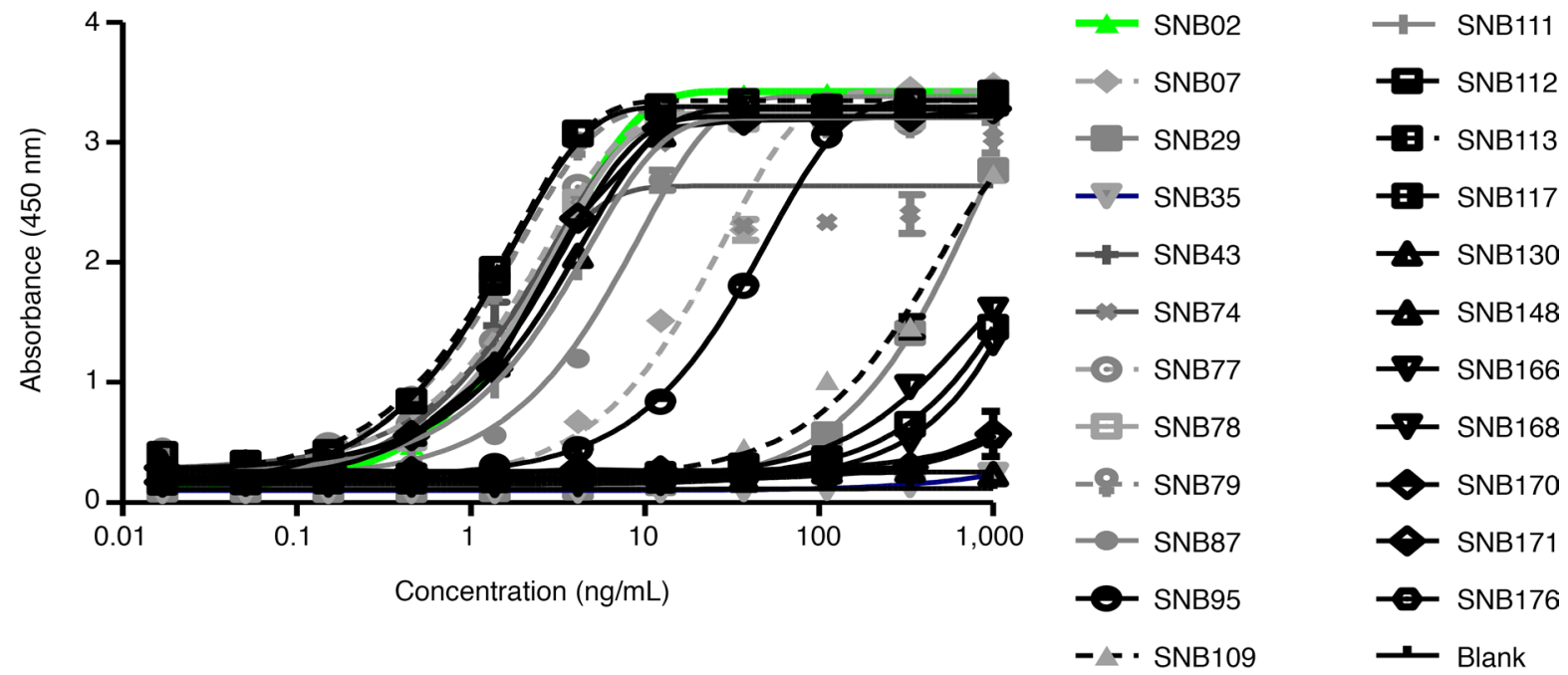

D
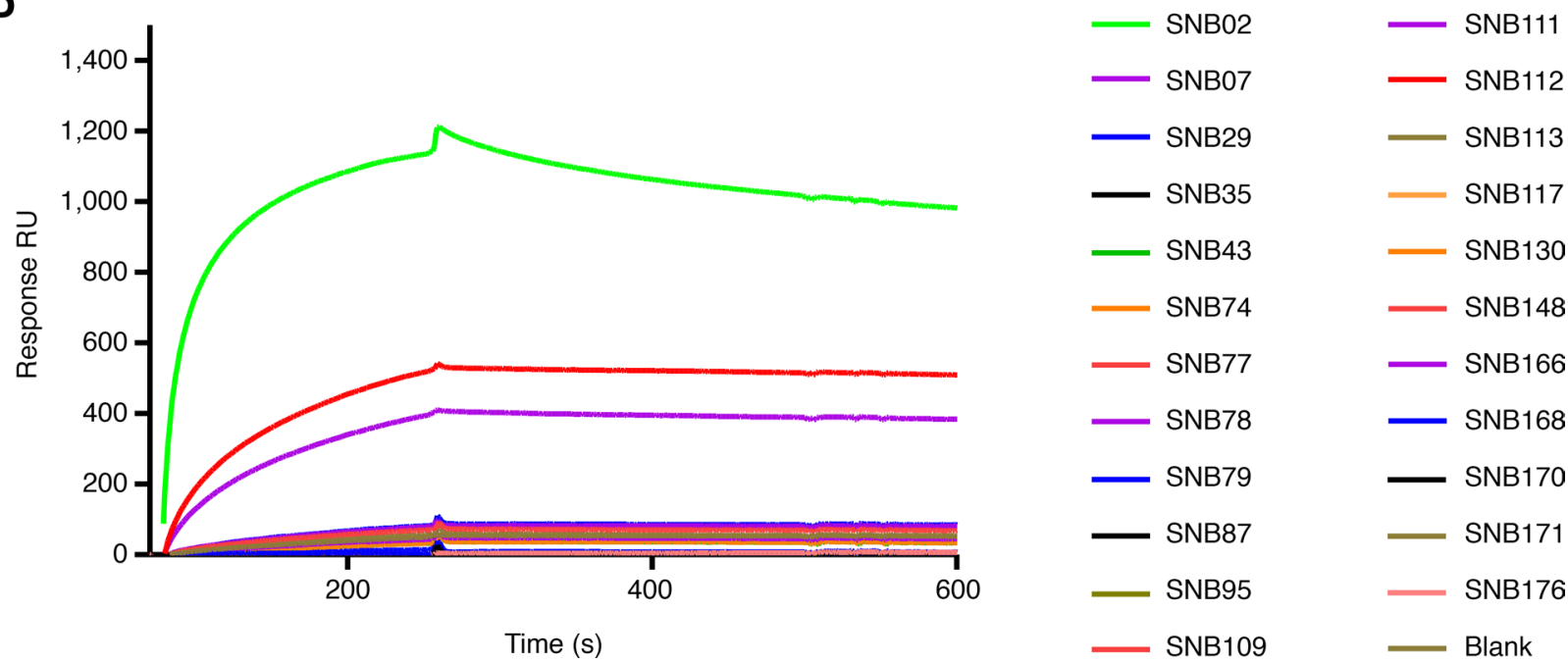

Figure 3. Design and characterization of SNB02. (A) Schematic diagram shows the structures of SNB, VHH + human Fc1 (CH2-3). (B) The graph shows the $\mathrm{IC}_{50}$ of 23 various SNBs, which were tested for neutralization against live SFTSV infection. The dashed line indicates that IC ${ }_{50}$ was more than $24 \mu \mathrm{g} / \mathrm{mL}$. Twenty-three various SNBs binding with sGn protein identified by ELISA (C) and by SPR (D). All experiments were repeated twice. 
A

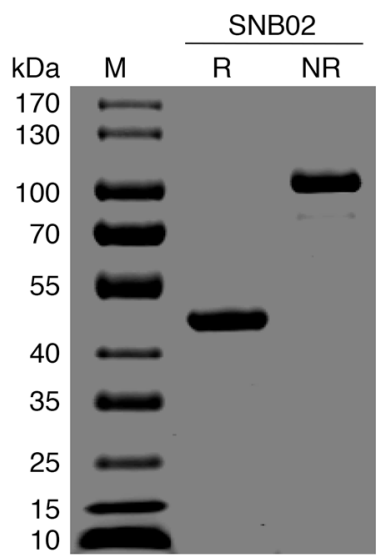

C

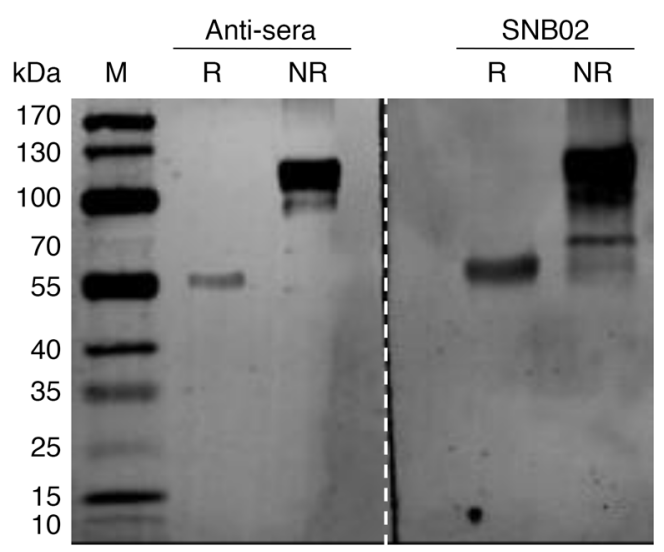

E

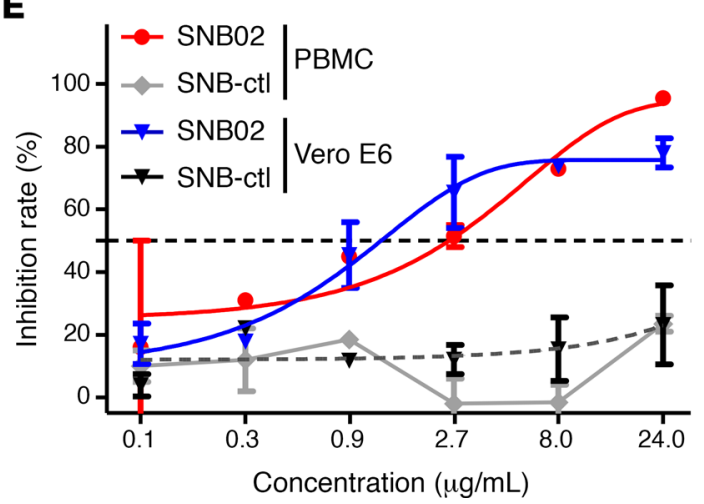

B

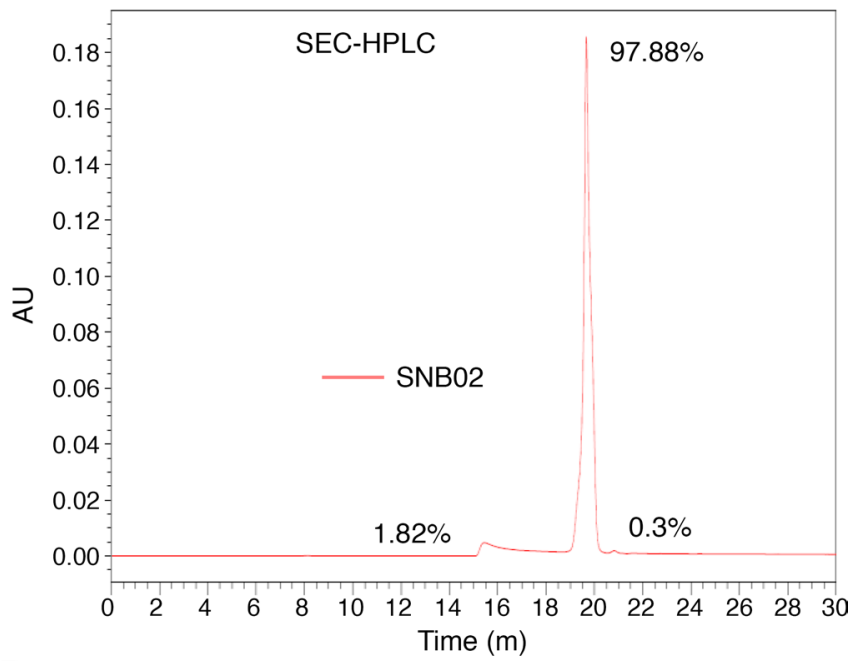

D

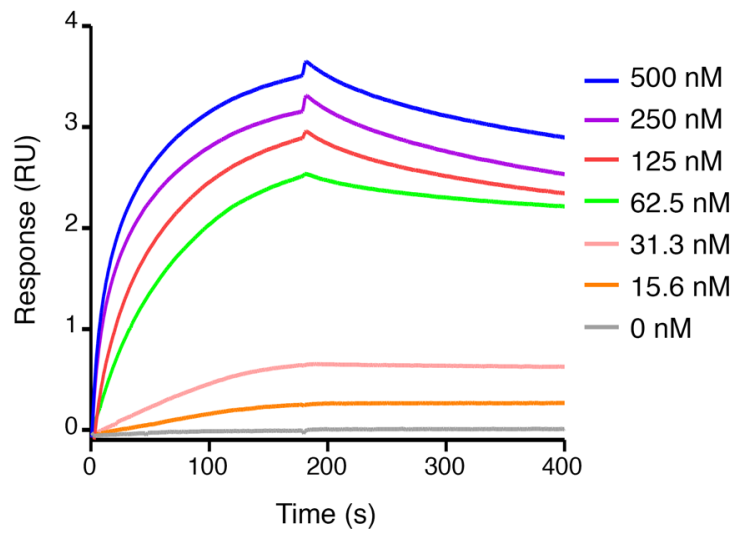

$\mathbf{F}$

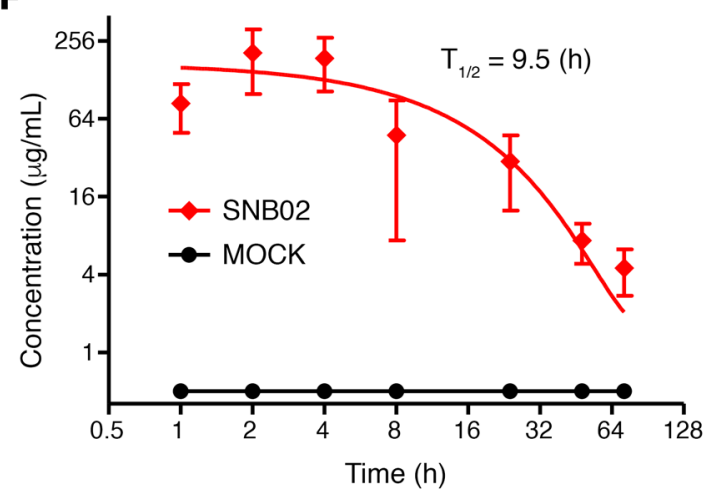

Figure 4. Characterization of SNBO2. (A) The purified SNBO2 was determined by SDS-PAGE under reducing condition (R) or nonreducing condition (NR). (B) Purity of SNBO2 was determined by size exclusion high-performance liquid chromatography (SEC-HPLC) analysis. (C) sGn under reducing conditions (R) or nonreducing conditions (NR) was detected by Western blot with SNBO2 and rabbit polyclonal antisera (Antisera) specific for sGn. (D) Kinetic binding curve of SNBO2 with sGn. Different-colored curves represent different concentrations; sGn protein was bound by SNBO2. (E) Neutralization activity of SNBO2 against live SFTSV infection of PBMCs and Vero E6 cells, respectively. SNB-ctl, 1 VHH-HuFc antibody without binding with sGn, was taken as antibody negative control. The red and gray lines show the neutralized curves of SNB02 and SNB-ctl inhibiting SFTSV to infect PBMCs, respectively. The blue line and the black lines show the neutralized curves of SNB02 and SNB-ctl inhibiting SFTSV to infect Vero E6 cells. (F) Bioavailability and $t_{1 / 2}$ of SNBO2 in NCG-HuPBL mice. Data represent mean \pm SEM. SNBO2 group, $n=4(2 \mathrm{M}+2 \mathrm{~F}) ;$ mock group, $n=2(1 \mathrm{M}+1 \mathrm{~F})$. All experiments of $\mathbf{A}, \mathbf{C}$, and $\mathbf{E}$ were repeated twice. 
A

SNB02 injection

$(400 \mu \mathrm{g} / \mathrm{d} \times 4$ d, i.p. $)$

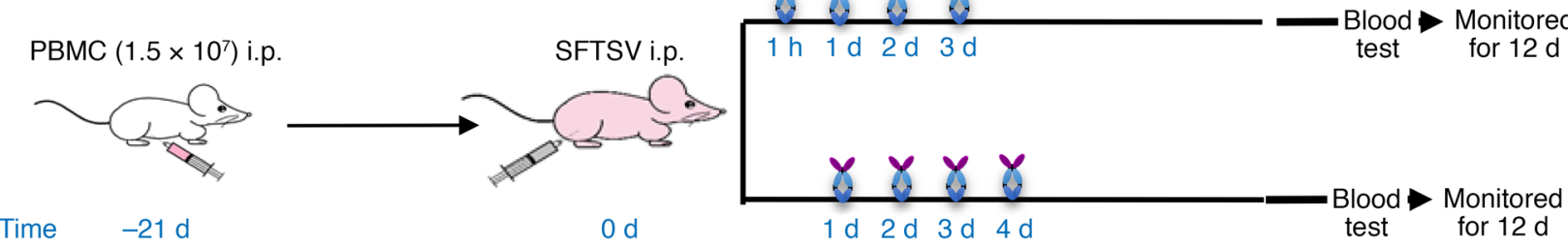

B

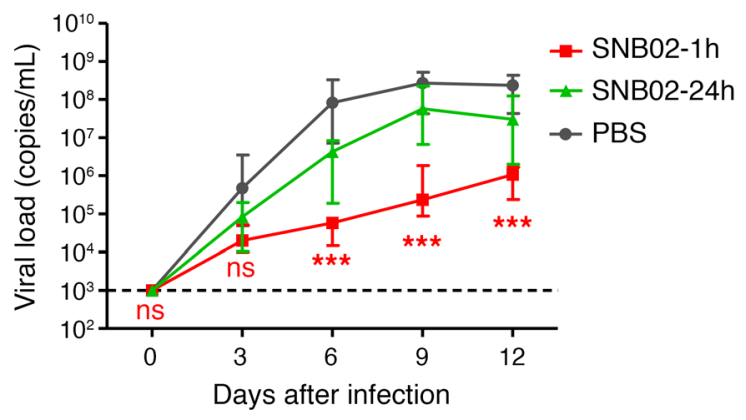

D

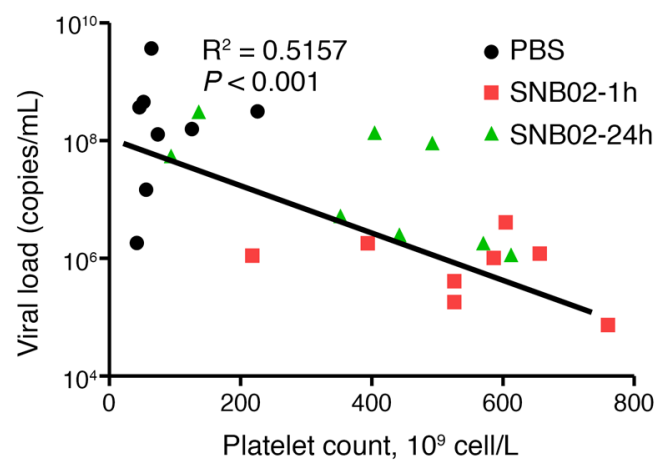

C

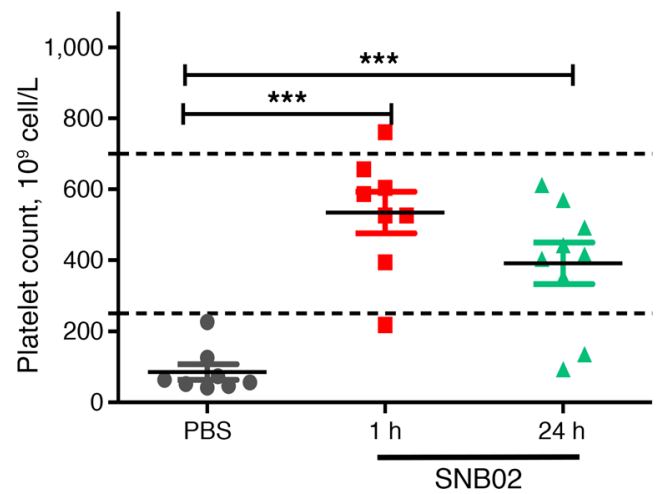

$\mathbf{E}$

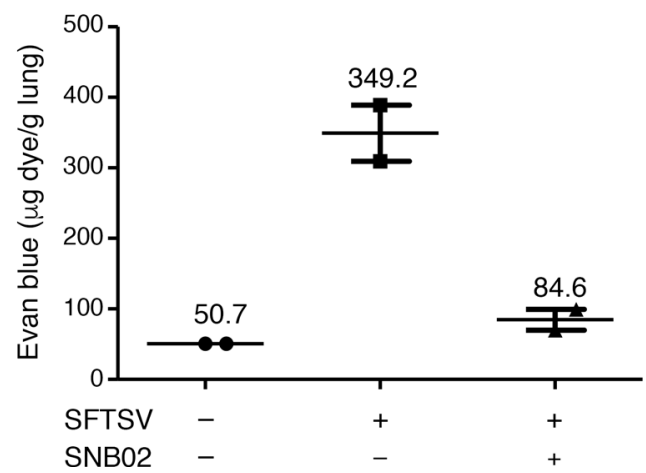

Figure 5. Protection of NCG-HuPBL mice against challenges of live SFTSV. (A) Experimental schedule of SNBO2 in the treatment of SFTSV infection. (B) Plasma viral loads among 3 groups of color-coded NCG-HuPBL mice including SFTSV challenge with PBS treatment as control (black, $n=8,4 \mathrm{M}+4 \mathrm{~F}$ ), SNB02 treatment 1 hour after SFTSV challenge (red, $n=8,4 \mathrm{M}+4 \mathrm{~F}$ ), and SNB02 treatment 1 day after SFTSV challenge (green, $n=84 \mathrm{M}+4 \mathrm{~F}$ ). Each line represents data from 1 group. Data represent median + range. The limit of detection was 1000 genome copies $/ \mathrm{mL}$. Two-way ANOVA with Tukey's test was performed to compare treatment group with control group. ns, no significance; ${ }^{* *} P<0.001$. Red ${ }^{* * *}$, the results of SNB02-1h compared with PBS control group; SNB02-24h compared with PBS had no significant difference. (C) Platelet cell counts were calculated among the same color-coded groups of animals. The normal range is between the 2 dashed lines. Each dot represents data from 1 mouse. Data represent mean \pm SEM; 1-way ANOVA with Bonferroni's multiple comparison was used for statistical analysis. (D) The correlation between plasma viral load and platelet counts at $12 \mathrm{dpi}$ among the same color-coded groups of animals. Correlation analyses were performed by linear regression using the GraphPad Prism 5.01 program. (E) Humanized SFTSV-infected NCG mice were treated with SNB02 or sham treatment. Humanized NCG mice without SFTSV infection were taken as a mock control. These mice were then given an intravenous injection of Evan's blue. Evan's blue accumulation was measured in lung to assess vascular permeability $(n=2)$. Data represent mean \pm SEM; 1-way ANOVA with Bonferroni's multiple comparison was used for statistical analysis. ns, no significance; ${ }^{* *} P<0.001$.

not suitable to evaluate in the aged ferret model resulting from the lack of human PBMCs to support antibody-dependent cellular cytotoxicity (ADCC) and/or antibody-dependent cellular phagocytosis (ADCP) by recognizing human Fc of humanized antibody. Given that humanized mice have been widely applied to evaluate the efficacy of human-specific virus infection, like HIV, dengue virus, Epstein-Barr virus, influenza, HBV, Ebola virus, Hantavirus, and so on (30), the humanized Hu-PBL mouse model was developed by us earlier for HIV research (31). For antibody efficacy evaluation, a humanized mouse model is particularly suited for a number of reasons. Virus-neutralizing antibodies carry out functions through 2 domains, the $\mathrm{VHH}$ or $\mathrm{ScFv}$ specifically binding with virus membrane proteins and interfering 
A WBC

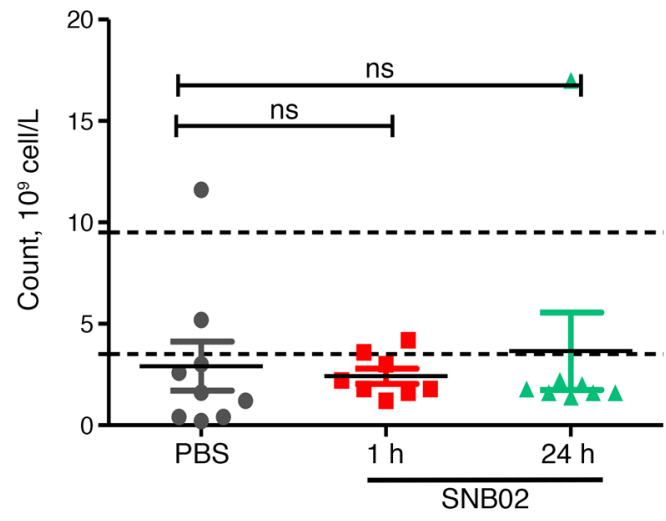

C Lymphocytes

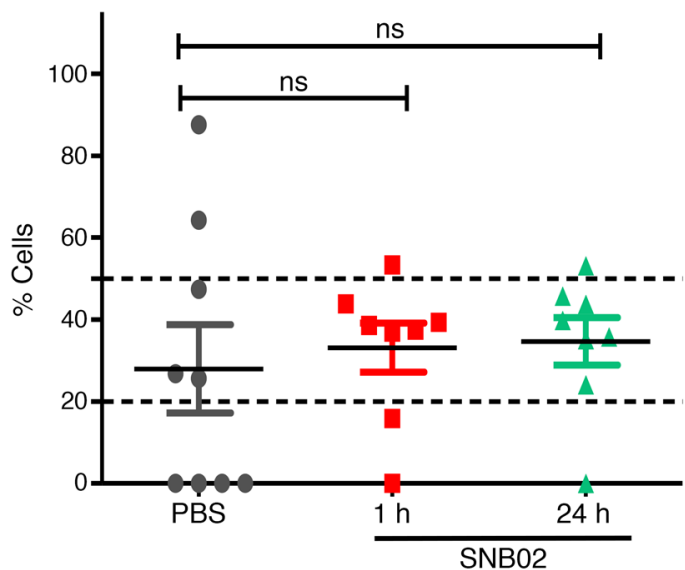

E Body weight

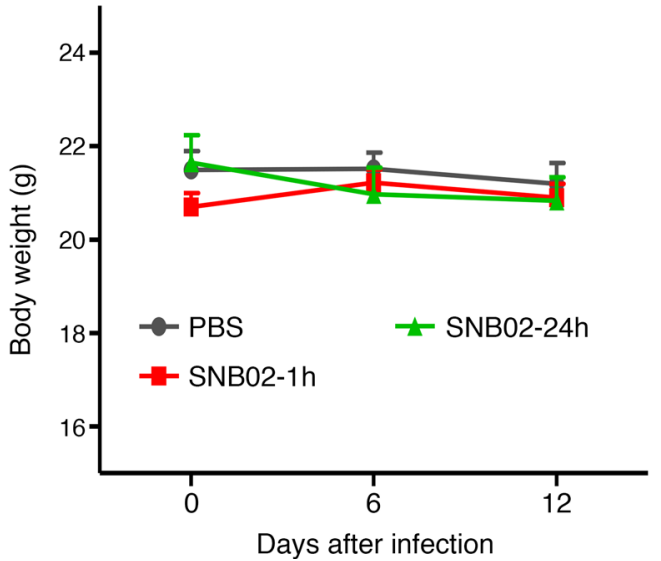

B Neutrophils

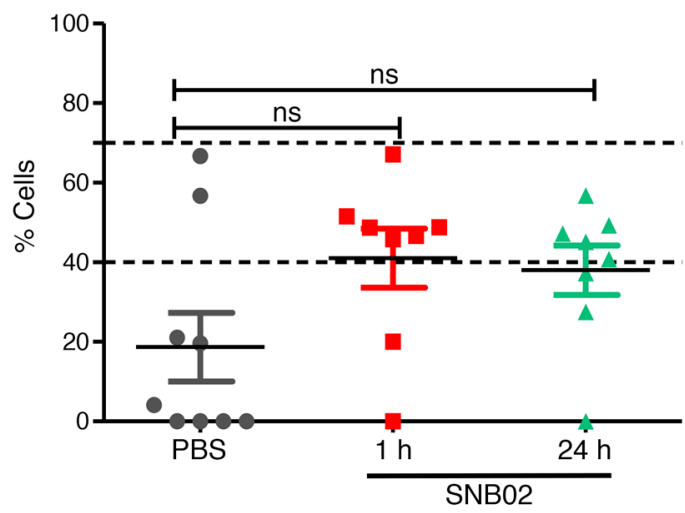

D Monocytes

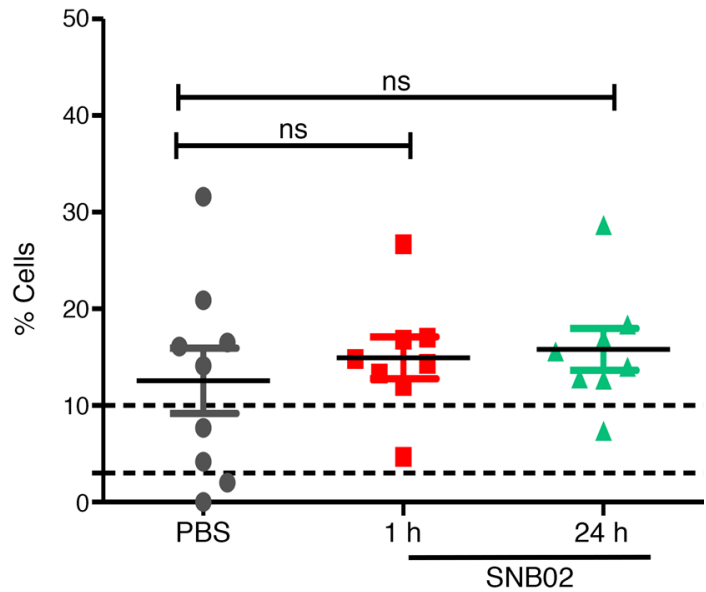

Figure 6. Evaluation of SNBO2 therapeutic efficacy in SFTSV-infected NCG-HuPBL mice. WBCs (A), neutrophils (B), lymphocytes (C), and monocytes (D) were analyzed in 3 groups of NCG-HuPBL mice infected with SFTSV and treated with or without SNBO2: SNBO2 treatment 1 hour after SFTSV challenge (red, $n=8,4 \mathrm{M}+4 \mathrm{~F}$ ), SNBO2 treatment 1 day after SFTSV challenge (green, $n=8,4 \mathrm{M}+4 \mathrm{~F}$ ). NCG-HuPBL mice infected with SFTSV but treated with PBS served as controls (black, $n=8,4 \mathrm{M}+4 \mathrm{~F}$ ). The normal range is between the 2 dashed lines. Each dot represents data from 1 mouse. (E) Body weight of mice among the above 3 groups. Each line represents data from 1 group. Data represent mean \pm SEM; 1-way ANOVA with Bonferroni's multiple comparison was used for statistical analysis. ns, no significance.

with viral entry to the cells, and the Fc domain playing other important roles, such as ADCC, ADCP, or even antibody-dependent enhancement (ADE). The Fc domain sometimes causes ADE by recruiting human macrophages and dendritic cells. ADE was commonly reported in the antibody treatment of dengue virus and SARS-CoV infection, which is a challenge for antibody drug development. Given this fact, 


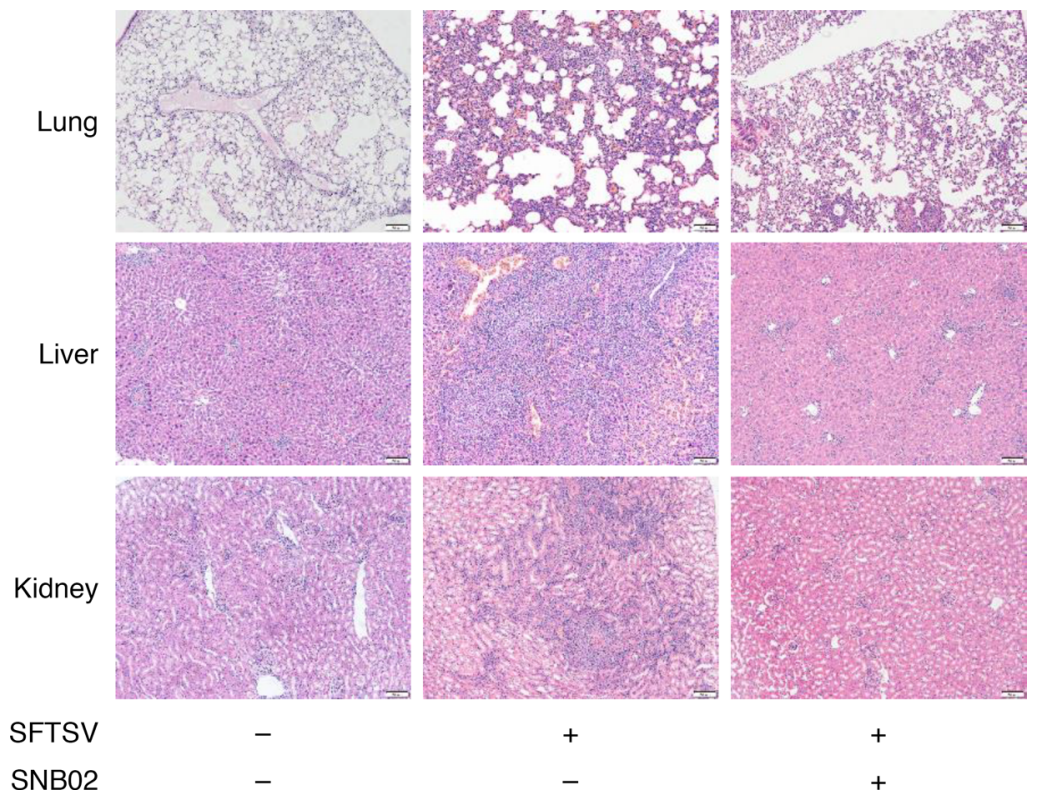

Figure 7. Representative sections of NCG-HuPBL mice were analyzed by H\&E staining. Tissue sections from mock NCG-HuPBL mice, SFTSV-infected NCG-HuPBL mice, and SFTSV-infected NCG-HuPBL mice receiving SNBO2 treatment are shown. Images were visualized under the $\times 10$ objective. All experiments were repeated twice.

Hu-PBL mice will be more suitable for evaluation of the efficacy and safety of SNB02, which carries a human $\mathrm{Fc}_{\mathrm{c}}$ at its $\mathrm{N}$-terminus, for the treatment of virus infection.

Our results also showed that SFTSV infected monocytes/macrophages in Hu-PBL mice as in humans and could replicate to high viral load and persist for several weeks. Moreover, the infected animals presented thrombocytopenia with a progressive decline in platelets. Graft-versus-host disease (GVHD) is a big challenge for Hu-PBL mice, but we can evaluate acute SFTSV infection before severe GVHD takes place, usually within 4-8 weeks. The infiltration of inflammatory cells in the lung, liver, and glomerular hyperemia in the kidney caused by SFTSV infection was observed (Figure 6), while significant fever and weight loss were not observed in SFTSV-infected Hu-PBL mice within 12 days postinfection (Figure 5E). The possible reason is that the time course of observation is not long enough. The Hu-PBL mouse model benefits from the robust $\mathrm{T}$ cell engraftment rather than that of mononuclear phagocytic cells (i.e., macrophages and dendritic cells) and B cells. But in Hu-PBL mice, there is also a small amount of monocytes/macrophages, which were infected by SFTSV, similar as in human infection. Given the above evidence, SFTSV infection of Hu-PBL NCG mice could recapitulate some major pathogenesis of SFTS in humans and present a useful model for investigating SFTSV-induced pathogenesis and evaluating antiviral agents, such as humanized antibodies.

Twenty-three VHH antibodies with potent neutralizing activities have been identified from our library. SNB02, a high-affinity antibody with a human Fc1, potently neutralized SFTSV in vitro with an $\mathrm{IC}_{50}$ of 1.05 $\mu \mathrm{g} / \mathrm{mL}$ and potently inhibited viral replication in a humanized mouse model. When SNB02 was applied 1 hour after viral inoculation, the viral titers were reduced by more than 2 logs for the entire 12-day experimental duration. This reduction may be significant because the differences of viral load between convalescent and dying patients as reported in clinical cohort studies were mostly within 2 logs $(32,33)$. However, we speculate that SNB02 would significantly improve its therapeutic efficacy if it were administrated in a 12-hour interval, with the half-life of the antibody being 9 hours. More importantly, the rebound of platelet counts suggests that SNB02 not only inhibits viral replication but also mitigates the virus-induced thrombocytopenia. Our NCG-HuPBL mouse data also showed that the disruption of blood vessel endothelium upon viral infection was markedly reversed by the antibody treatment, demonstrating that SNB02 also lessens the virus-induced pathogenesis. The time-lapse analysis of the antibody treatment clearly indicated that the efficacy of SNB02 will diminish if the antibody is administrated 1 day or longer after infection of NCG-HuPBL mice. This is likely because SFTSV establishes robust infection almost immediately upon viral entry and replicates to high titer within 3 days in NCG-HuPBL mice (Figure 5B). During natural infection, the peak viremia is usually detected on days $3-5$ from the onset of symptoms, indicating that virus spreading 
to organs would happen rapidly, and it is critical to control viremia at the early stage. Viral replication and host immune responses affect the severity and clinical outcome of SFTS $(33,34)$. Comparison of laboratory measurements between fatal and nonfatal cases has shown that high blood viral RNA load of 10 copies $/ \mathrm{mL}$ or more are associated with death (2).

There are a number of limitations in the current study, and extrapolating the observations into human use should proceed with caution (1). The clinical course of the disease in NCG-HuPBL mice is distinct from that of humans. Therefore, the optimal application of the antibody postinfection requires validation in human tests; though NCG-HuPBL mouse study suggested early intervention (2), the humanized SNB02 may have a rather different degradation kinetics in mice than humans. For a better evaluation, a nonhuman primate model, which we are currently developing, may offer a more relevant insight into the antibody half-life and its in vivo activity (3) The relatively short half-life of SNB02 observed in Hu-PBL mice can be improved by a number of approaches, including fusion with optimized Fc, polyethylene glycol (PEG), or another VHH specific for human serum albumin (35).

Nanobody has recently been studied for various applications because of its high solubility, thermostability, low toxicity, and low immunogenicity. Nanobody drugs for tumors, inflammation, infectious diseases, and neurological diseases are in various stages of development (15). Given that patients infected by SFTSV are mainly from rural areas, the high thermostability of nanobodies is an advantage for non-cold chain transportation and storage. Because nanobodies from camelids are generally not highly immunogenic in humans, it may not be necessary to carry out humanization of the nanobody for human use, though humanization of SNB02 showed no impact on its binding and neutralization activities (Supplemental Figure 7). VHH-derived SNB02 is thus a promising candidate as a therapeutic antibody for the treatment of SFTSV infection.

\section{Methods}

Camel immunization. First, $250 \mu \mathrm{g}$ sGn-rFc protein was emulsified with $250 \mu \mathrm{L}$ Freund's complete adjuvant (F5881-10ML, MilliporeSigma) to immunize a bimodal camel. On days 14, 28, and 42, the camel was boosted with $250 \mu \mathrm{g}$ sGn-rFc protein in $250 \mu \mathrm{L}$ Freund's incomplete adjuvant (F5506-10ML, MilliporeSigma) 3 times. One week after the second and third immunizations, blood samples were collected for antiserum titer measurement; 1 week after the fourth immunization, $100 \mathrm{~mL}$ of blood was collected for the construction of a phage library displaying $\mathrm{VHH}$ antibody.

SDS-PAGE and Western blotting. The protein, engineered with either a His-tag or an $\mathrm{rFc}$ tag at the N-terminus, was expressed by transiently transfecting FreeStyle 293-F cells (R79007, Thermo Fisher Scientific) and purified from the cell culture supernatant using either commercial Ni-NTA (R901100, Thermo Fisher Scientific) or Protein A Sepharose (20334, Thermo Fisher Scientific), respectively. Purified protein or antibody was separated by electrophoresis in a $7.5 \%-12 \%$ polyacrylamide gel. The separated protein or antibody was revealed by Coomassie blue or transferred to a PVDF membrane. The reducing and nonreducing conditions refer to the presence and absence of $\beta$-mercaptoethanol in the gel-loading buffer. The membrane was first blocked and then incubated at $4^{\circ} \mathrm{C}$ overnight or $37^{\circ} \mathrm{C}$ for 1 hour with the diluted plasma or antibody, followed by a secondary antibody of either anti-human IgG or anti-rabbit IgG with an IRDye 800CW (926-32232, Rockland). Protein bands were visualized using Odyssey CLx Imaging System (LI-COR).

ELISA for characterizing antisera and antibody. Antisera titer and antibody characterization were assessed by ELISA according to our previously published protocols (36) with the following modifications. Briefly, protein was coated to high-protein-binding ELISA plates (9018, Corning) at a concentration of $0.5 \mu \mathrm{g} / \mathrm{mL}$, $100 \mu \mathrm{L} /$ well, at $37^{\circ} \mathrm{C}$ for 2 hours or $4^{\circ} \mathrm{C}$ overnight. After washing, blocking buffer with $5 \%$ nonfat milk in PBS was added and incubated at $37^{\circ} \mathrm{C}$ for 1 hour. After washing 2-4 times, $100 \mu \mathrm{L}$ serially diluted antisera or purified antibody was added and incubated at $37^{\circ} \mathrm{C}$ for 1.5 hours. After washing, goat anti-1lama IgG $(\mathrm{H}+\mathrm{L})$ secondary antibody with $\mathrm{HRP}\left(1: 10,000\right.$ dilution, NB7242, Novus) was added and incubated at $37^{\circ} \mathrm{C}$ for 1 hour. Accordingly, 3,3',5,5'-tetramethylbenzidine (TMB, MilliporeSigma) substrate was added at $37^{\circ} \mathrm{C}$ for 10 minutes; $10 \mu \mathrm{L} 0.2 \mathrm{M} \mathrm{H}_{2} \mathrm{SO}_{4}$ was added to stop the reaction. ODs were measured at $450 \mathrm{~nm}$ using the Infinite 200 (Tecan). Antibody titers were characterized as the highest dilution where the diluted sample produced at least 2.1-fold greater OD readout than that of the control serum sample at the same dilution.

Immunofluorescence analysis. M gene encoding SFTSV Gn and Gc protein from various genotypes of A-Gongwon Korea 2012, B-HB29, C-SD4, D-JS3, and E-KAGWH3 were obtained from the National Center for Biotechnology Information's website (37). M genes were synthesized and constructed in pCAGGS 
by GenScript as expression plasmids. The plasmids were transfected into 293TT cells (donated by Y-Clone) cultured in 12-well plates. Then, 48 hours posttransfection, the cells were washed by PBS and fixed with $4 \%$ paraformaldehyde for 15 minutes at room temperature. The antisera of the immunized camel (1:1500) was used to stain the 293TT cells, followed by Alexa Fluor 488 AffiniPure goat anti-alpaca IgG (H+L) (1:500 dilution) (128-545-160, Jackson ImmunoResearch). The images were acquired using an OLYMPUS IX73.

Construction of a phage library displaying VHH antibody. A VHH phage library was constructed as previously reported with the following modifications (38). Briefly, PBMCs were isolated from $100 \mathrm{~mL}$ blood from the immunized camel using a lymphocyte separation solution (GE Ficoll-Paque Plus, 17-1140-02). According to the TRIzol kit instructions, RNA was extracted and reverse-transcribed into cDNA by oligo (dT) and random hexamers as primers. The camel VHH gene was amplified with the combination of primers and cloned into phV1 phagemid plasmid (Y-Clone) to transform TG1 bacteria.

Panning VHH phage library. Affinity selection for Gn-binding recombinant phages was performed as previously reported with the following modifications (39). The VHH-phagemid-transformed bacteria were rescued with the help of M13KO7 helper phage (18311019, Invitrogen, Thermo Fisher Scientific) and precipitated with PEG/NaCl. The phage VHH antibody library was enriched 3 times with $50 \mu \mathrm{g} / \mathrm{mL}$ of sGn-His protein. The enriched phage was eluted, transformed, and picked for monoclonal to be assessed by phage ELISA using sGn protein as antigen. For phage ELISA, 200 ng sGn-His protein in coating buffer (pH 9.6) was used to coat 96 -well plates (9018, Corning) at $4^{\circ} \mathrm{C}$ overnight. After washing, the plates were blocked for 1 hour at $37^{\circ} \mathrm{C}$ with blocking buffer (3\% BSA in PBS-Tween; PBST) and then incubated with library phages or a single-clone phage in bacterial supernatant at $4^{\circ} \mathrm{C}$ for 1.5 hours. After washing, anti-M13 bacteriophage antibody with HRP (1:10,000 dilution, 11973-MM05T-H, Sino Biological) was added and incubated at $37^{\circ} \mathrm{C}$ for 1 hour. Accordingly, TMB substrate was added at $37^{\circ} \mathrm{C}$ for 10 minutes; $10 \mu \mathrm{L} 0.2 \mathrm{M} \mathrm{H}_{2} \mathrm{SO}_{4}$ was added to stop the reaction. ODs were measured at $450 \mathrm{~nm}$ using the Infinite 200. Clones with readout at 450 $\mathrm{nm}$ more than 0.5 were sequenced.

Construction and expression of VHH antibody library. VHH gene fragments were purified after PCR amplification of the enriched phage $\mathrm{VHH}$ antibody library, which was cloned into a prokaryotic expression vector, pComb3X (63891, Addgene). VHH gene fragment-containg vectors were transformed into SS320 cells to produce a prokaryotic expression VHH antibody library. This bacterial VHH antibody library was spread on a plate, and 1399 clones were randomly selected on the next day for evaluation. The bacteria were induced to be expressed by isopropyl $\beta$-D-thiogalactopyranoside, and then the bacterial supernatant was analyzed by ELISA with sGn protein as antigen; all (sGn/blank at $450 \mathrm{~nm}$ ) ratios greater than 5.0 were selected as positive clones. The corresponding bacterial clone was sent for sequencing. The supernatant of the positive clone was accessed by neutralization assay against SFTSV infection.

SNB eukaryotic expression. In order to facilitate the purification and to prolong the half-life of the VHH antibody, the $\mathrm{Fc1}$ gene ( $\mathrm{CH} 2-3)$ of human monoclonal antibody was fused with $\mathrm{VHH}$ gene to be cloned into the pVAX1 eukaryotic expression vector. The constructed VHH-huFc-pVAX1 was transfected into 293-F cells to produce VHH-huFc (SNB) antibody, which was purified using Protein G (20399, Thermo Fisher Scientific). SNB02 was constructed and produced with the same method based on the P88 VHH clones (Supplemental Table 3).

SPR analysis. SPR was conducted according to our published protocols with the following modifications (31). Four thousand response units of sGn protein was coupled to the CM5 chip via a BIAcore X100 instrument (GE Healthcare) assisted with Bio-evaluation version 4.0 according to the instructions of the amino coupling kit, and ethanolamine was used to block the coupled chip. Antibodies were diluted to different concentrations and used in the mobile phase. Antibody concentration was used from low to high with 2 repeated analyses per concentration; binding time was set to 180 seconds, flow rate of $30 \mu \mathrm{L} / \mathrm{min}$; dissociation time was set to 180 seconds, flow rate at $30 \mu \mathrm{L} / \mathrm{min} ; \mathrm{pH} 2.5 ; 10 \mathrm{mM}$ glycine was set to $30 \mu \mathrm{L} /$ min for 30 seconds to activate and regenerate the surface of the chip; PBS was equilibrated for 5 seconds at a flow rate of $30 \mu \mathrm{L} / \mathrm{min}$. The experimental data were analyzed to calculate binding, dissociation, and affinity constants. Binding curves were plotted after the subtraction of backgrounds. The 2:1 bivalent analyte model was used for the calculations according to the instructions.

VHH or SNB neutralizes SFTSV-infected PBMCs and Vero E6 cells. Neutralization was conducted as previously described (14). Briefly, SFTSV of subtype E-JS-2013-24 was provided by Jiangsu Provincial CDC, Nanjing, China. The virus was passaged in Vero E6 cells. VHH antibody, SNB antibody, or camel antiserum was serially diluted and incubated with 1000 tissue culture $\mathrm{ID}_{50}\left(\mathrm{TCID}_{50}\right)$ SFTSV of subtype E-JS-2013-24 
at $5 \% \mathrm{CO}_{2} 37^{\circ} \mathrm{C}$ for 1 hour in 96-well plates. $1.5 \times 10^{4}$ Vero E6 cells were added and incubated for 48 hours at $5 \% \mathrm{CO}_{2} 37^{\circ} \mathrm{C}$. After removing the cell supernatant, the cells were fixed with $4 \%$ paraformaldehyde for 15 minutes. After washing and blocking, anti-Gn rabbit polyclonal serum (Y-Clone) was added at 1:1000 dilution and incubated overnight at $4^{\circ} \mathrm{C}$. After washing with PBST, $50 \mu \mathrm{L}$ of anti-rabbit secondary antibody, Alexa Fluor 488 anti-rabbit IgG (H+L) (111-545-144, Jackson ImmunoResearch Laboratories), was added at 1:1000 dilution and incubated for 40 minutes at room temperature. Images were obtained by OLYMPUS IX73 using HCImage Live $(\times 64)$ software and analyzed by ImageJ $(\mathrm{NIH})$. Inhibition rate $=(1-$ average of fluorescence intensity of sample group - mean fluorescence intensity of cell-only control of cell control group]/[mean of fluorescence intensity of control treatment group - mean fluorescence intensity of cell-only control of cell control group]) $\times 100 \%$. The neutralization titer $\left(\mathrm{ID}_{50}\right.$ or $\left.\mathrm{IC}_{50}\right)$ is expressed as a multiple of the dilution at $50 \%$ inhibition.

The neutralization of SNB against SFTSV-infected PBMCs was conducted similarly as Vero E6 cells. In brief, SNB antibody was serially diluted and incubated with $6000 \mathrm{TCID}_{50}$ SFTSV of subtype E-JS-201324 at $5 \% \mathrm{CO}_{2} 37^{\circ} \mathrm{C}$ for 1 hour in 12 -well plates. $8.0 \times 10^{6} \mathrm{PBMCs}$ were added and incubated for 48 hours at $5 \% \mathrm{CO}_{2}$ at $37^{\circ} \mathrm{C}$. After removing the cell supernatant, SFTS viral titer was determined in the pelleted cells by quantitative reverse transcription PCR (RT-PCR). The neutralization titer $\left(\mathrm{ID}_{50}\right.$ or $\left.\mathrm{IC}_{50}\right)$ is expressed as a multiple of the dilution at $50 \%$ inhibition. Inhibition rate $=(1-$ average of viral copy number of sample group - mean viral copy number of CC of cell control group]/[mean of viral copy number of control treatment group - mean viral copy number of $\mathrm{CC}$ of cell control group]) $\times 100 \%$. The neutralization titer $\left(\mathrm{ID}_{50}\right.$ or $\mathrm{IC}_{50}$ ) is expressed as a multiple of the dilution at $50 \%$ inhibition.

In vivo evaluation of SNB inhibition of SFTSV infection in NCG mice. Immunodeficient NCG mice were

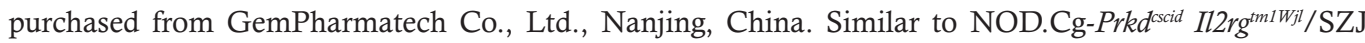
mice, the NCG mice lacked the IL2 receptor gene in a SCID background, resulting in the absence of murine $\mathrm{T}$ and $\mathrm{B}$ cells and very small numbers of NK cells $(40) .1 .0 \times 10^{6}$ to $1.5 \times 10^{7}$ human PBMCs (HuPBL) were injected i.p. into 4- to 6-week-old NCG mice; 3 weeks later, blood samples from NCG-HuPBL mice were subjected to flow cytometry to determine the percentage of human $\mathrm{CD} 45^{+}, \mathrm{CD}^{+}, \mathrm{CD}^{+}$, and $\mathrm{CD} 8^{+}$. If the proportion of human CD45 $5^{+}$cells reached more than $5 \%$, NCG-HuPBL mice were challenged with $2 \times 10^{5} \mathrm{TCID}_{50}$ of SFTSV of subtype E-JS-2013-24 (37). The infected NCG-HuPBL mice $(n=8,4 \mathrm{M}$ and 4 F) were treated with $400 \mu \mathrm{g}$ mouse SNB02 i.p. 1 hour, 1 day, 2 days, and 3 days postinfection or 1 day, 2 days, 3 days, and 4 days postinfection. Infected mice treated with PBS on the same time interval served as control. On days $0,3,6,9$, and 12 , the blood was collected for monitoring viral load by quantitative PCR or for completing blood count conducted at Gulou Hospital (Jiangsu, China).

Evan's blue permeability and tissue histology. Vascular permeability in the lung was assessed with Evan's blue as previously reported with the following modifications (41). NCG-HuPBL mice were challenged with $2 \times 10^{5} \mathrm{TCID}_{50}$ of SFTSV. The infected NCG-HuPBL mice $(n=2,1 \mathrm{M}$ and $1 \mathrm{~F})$ were treated with 400 $\mu \mathrm{g} /$ mouse SNB02 i.p. 1 hour, 1 day, 4 days, and 7 days postinfection. Infected mice treated with PBS on the same time interval served as negative control. NCG-HuPBL mice without SFTSV infection served as mock control. Eight days after SFTSV infection, mice were given an intravenous injection of Evan's blue (40 mg/kg, Macklin, E6135-25g). Evan's blue was allowed to circulate for 1 hour, and the mice were deeply anesthetized and perfused with saline plus $5 \mathrm{mM}$ EDTA. Lungs were excised, weighed, and homogenized in $2 \mathrm{~mL}$ of PBS. $4 \mathrm{~mL}$ Formamide (Invitrogen, Thermo Fisher Scientific) was added. The samples were incubated overnight at $60^{\circ} \mathrm{C}$ and then centrifuged at $800 \mathrm{~g}$ for 5 minutes to extract Evan's blue dye. The samples were then centrifuged, and supernatants were analyzed by spectrophotometry at both 620 and 740 $\mathrm{nm}$. The absorbance was normalized as described (41) and converted to micrograms Evan's blue dye per gram wet weight of lungs. Data are presented as mean \pm SEM. After mice was sacrificed, $4-\mu m$-thick paraffin-embedded tissue sections were prepared for H\&E staining by Gulou Hospital.

Viral load measurement by quantitative RT-PCR. Viral RNA was extracted using TRIzol reagent (15596018 Thermo Fisher Scientific). Each RNA sample was reverse-transcribed to $10 \mu \mathrm{L}$ cDNA with PrimeScript RT Master Mix (036A Takara). cDNA $(2 \mu \mathrm{L})$ was used in a $20-\mu \mathrm{L}$ quantitative RT-PCR reaction with PowerUp SYBR Green Master Mix (A25742 Life Technologies, Thermo Fisher Scientific). Primers were designed to target the L gene of SFTSV (5'-TGGTGGATGTCATAGAAGGC-3' and 5'-GAGTAATCCTCTTGCCTGCT-3'). The samples were run in triplicate on an ABI 7500 Real-Time System (Applied Biosystems, Thermo Fisher Scientific). The following cycling conditions were used: 1 cycle of $50^{\circ} \mathrm{C}$ for 2 minutes, 1 cycle of $95^{\circ} \mathrm{C}$ for 10 minutes, and 40 cycles of $95^{\circ} \mathrm{C}$ for 15 seconds and $60^{\circ} \mathrm{C}$ for 1 minute. The virus titer 
was determined by comparison with a standard curve generated using a plasmid (synthesized by Synbio Technologies) from a serial dilution. The limit of detection was about 1000 SFTSV copies $/ \mathrm{mL}$.

Statistics. Graphs were generated by GraphPad Prism 5.01 software or OriginPro 8.5 software (OriginLab). One- or 2-way ANOVA was performed for group comparisons. $P<0.05$ was considered statistically significant with data shown as mean \pm SEM or mean \pm SD or median + range.

Study approval. The study and the protocol for this research were approved by the Center for Public Health Research, Medical School, Nanjing University. All animal experimental procedures were approved by the Committee on the Use of Live Animals by the Ethics Committee of Nanjing Drum Tower Hospital. All the authors declare their compliance with publishing ethics.

\section{Author contributions}

$\mathrm{XW}$ and YL conducted most experiments, analyzed the data, and wrote the manuscript. XM, LZ, BH, $\mathrm{NZ}, \mathrm{SX}, \mathrm{WN}$, and CX provided technical assistance and did animal experiments. ZW designed the study, monitored and financially supported the study, and revised the manuscript. All authors critically reviewed the manuscript and approved the final version.

\section{Acknowledgments}

This work was supported by National Science Foundation of China (81803414, 31970149), The Major Research and Development Project (2018ZX10301406), Nanjing University-Ningxia University Collaborative Project (2017BN04), Jiangsu Province Natural Science Foundation for Young Scholar (BK20170653), Jiangsu province "Innovative and Entrepreneurial talent," and Six Talent Peaks Project of Jiangsu Province.

Address correspondence to: Zhiwei Wu, School of Life Sciences, Ningxia University, 539 Helanshan Road West, Yinchuan 750021, China. Phone: 86.25.8368.6092; Email: wzhw@nju.edu.cn.

1. Yu XJ, et al. Fever with thrombocytopenia associated with a novel bunyavirus in China. N Engl J Med. 2011;364(16):1523-1532.

2. Liu Q, He B, Huang SY, Wei F, Zhu XQ. Severe fever with thrombocytopenia syndrome, an emerging tick-borne zoonosis. Lancet Infect Dis. 2014;14(8):763-772.

3. Fang LQ, et al. Emerging tick-borne infections in mainland China: an increasing public health threat. Lancet Infect Dis. 2015;15(12):1467-1479.

4. Guo CT, et al. Epidemiological and clinical characteristics of severe fever with thrombocytopenia syndrome (SFTS) in China: an integrated data analysis. Epidemiol Infect. 2016;144(6):1345-1354.

5. Tang $X$, et al. Human-to-human transmission of severe fever with thrombocytopenia syndrome bunyavirus through contact with infectious blood. J Infect Dis. 2013;207(5):736-739.

6. Gai Z, et al. Person-to-person transmission of severe fever with thrombocytopenia syndrome bunyavirus through blood contact. Clin Infect Dis. 2012;54(2):249-252.

7. Lee SY, et al. Complete genome sequences of two severe fever with thrombocytopenia syndrome virus strains isolated from a human and a dog in the Republic of Korea. Microbiol Resour Announc. 2019;8(31):e01695-18.

8. Tabara K, Fujita H, Hirata A, Hayasaka D. Investigation of severe fever with thrombocytopenia syndrome virus antibody among domestic bovines transported to slaughterhouse in Shimane Prefecture, Japan. Jpn J Infect Dis. 2016;69(5):445-447.

9. Niu G, et al. Severe fever with thrombocytopenia syndrome virus among domesticated animals, China. Emerging Infect Dis. 2013;19(5):756-763.

10. Robles NJC, Han HJ, Park SJ, Choi YK. Epidemiology of severe fever and thrombocytopenia syndrome virus infection and the need for therapeutics for the prevention. Clin Exp Vaccine Res. 2018;7(1):43-50.

11. Huang YT, Zhao L, Wen HL, Yang Y, Yu H, Yu XJ. Neutralizing antibodies to severe fever with thrombocytopenia syndrome virus 4 years after hospitalization, China. Emerging Infect Dis. 2016;22(11):1985-1987.

12. Wu Y, et al. Structures of phlebovirus glycoprotein $\mathrm{Gn}$ and identification of a neutralizing antibody epitope. Proc Natl Acad Sci U $S$ A. 2017;114(36):E7564-E7573.

13. Shi X, Kohl A, Li P, Elliott RM. Role of the cytoplasmic tail domains of Bunyamwera orthobunyavirus glycoproteins Gn and Gc in virus assembly and morphogenesis. J Virol. 2007;81(18):10151-10160.

14. Song P, et al. Deficient humoral responses and disrupted B-cell immunity are associated with fatal SFTSV infection. Nat Com mun. 2018;9(1):3328.

15. Muyldermans S. Nanobodies: natural single-domain antibodies. In: Kornberg RD, ed. Annual Review of Biochemistry. 2013;82:775-797.

16. Harmsen MM, De Haard HJ. Properties, production, and applications of camelid single-domain antibody fragments. Appl Microbiol Biotechnol. 2007;77(1):13-22.

17. Bannas P, Hambach J, Koch-Nolte F. Nanobodies and nanobody-based human heavy chain antibodies as antitumor therapeutics. Front Immunol. 2017;8:1603.

18. Steeland S, Vandenbroucke RE, Libert C. Nanobodies as therapeutics: big opportunities for small antibodies. Drug Discov Today. 2016;21(7):1076-1113. 
19. Kaplon H, Reichert JM. Antibodies to watch in 2019. MAbs. 2019;11(2):219-238.

20. Xu J, et al. A phase I, open, multiple dose, dose escalation and expansion study to evaluate the safety, tolerability, and pharmacokinetics of KN035 (anti-PD-L1 antibody) administered in subcutaneous injection as a single agent to Chinese subjects with advanced solid tumors. J Clin Oncol. 2018;36(Suppl 15):e15138.

21. Chen C, et al. Animals as amplification hosts in the spread of severe fever with thrombocytopenia syndrome virus: a systematic review and meta-analysis. Int J Infect Dis. 2019;79:77-84.

22. Chen H, Hu K, Zou J, Xiao J. A cluster of cases of human-to-human transmission caused by severe fever with thrombocytopenia syndrome bunyavirus. Int J Infect Dis. 2013;17(3):e206-e208.

23. Zhao L, et al. Severe fever with thrombocytopenia syndrome virus, Shandong Province, China. Emerging Infect Dis. 2012;18(6):963-965

24. Rainey T, Occi JL, Robbins RG, Egizi A. Discovery of Haemaphysalis longicornis (Ixodida: Ixodidae) parasitizing a sheep in New Jersey, United States. J Med Entomol. 2018;55(3):757-759.

25. Guo X, et al. Human antibody neutralizes severe Fever with thrombocytopenia syndrome virus, an emerging hemorrhagic Fever virus. Clin Vaccine Immunol. 2013;20(9):1426-1432.

26. Kim KH, et al. An anti-Gn glycoprotein antibody from a convalescent patient potently inhibits the infection of severe fever with thrombocytopenia syndrome virus. PLoS Pathog. 2019;15(2):e1007375.

27. Jin C, et al. Pathogenesis of emerging severe fever with thrombocytopenia syndrome virus in C57/BL6 mouse model. Proc Natl Acad Sci U S A. 2012;109(25):10053-10058.

28. Liu Y, Wu B, Paessler S, Walker DH, Tesh RB, Yu XJ. The pathogenesis of severe fever with thrombocytopenia syndrome virus infection in alpha/beta interferon knockout mice: insights into the pathologic mechanisms of a new viral hemorrhagic fever. J Virol. 2014;88(3):1781-1786.

29. Park SJ, et al. Ferret animal model of severe fever with thrombocytopenia syndrome phlebovirus for human lethal infection and pathogenesis. Nat Microbiol. 2019;4(3):438-446.

30. Walsh NC, et al. Humanized mouse models of clinical disease. Annu Rev Pathol. 2017;12:187-215.

31. Wu X, et al. Tandem bispecific neutralizing antibody eliminates HIV-1 infection in humanized mice. J Clin Invest. 2018;128(6):2239-2251.

32. Li H, et al. Epidemiological and clinical features of laboratory-diagnosed severe fever with thrombocytopenia syndrome in China, 2011-17: a prospective observational study. Lancet Infect Dis. 2018;18(10):1127-1137.

33. Li DX. Severe fever with thrombocytopenia syndrome: a newly discovered emerging infectious disease. Clin Microbiol Infect. 2015;21(7):614-620.

34. Liu S, et al. Systematic review of severe fever with thrombocytopenia syndrome: virology, epidemiology, and clinical characteristics. Rev Med Virol. 2014;24(2):90-102.

35. De Vlieger D, Ballegeer M, Rossey I, Schepens B, Saelens X. Single-domain antibodies and their formatting to combat viral infections. Antibodies (Basel). 2018;8(1):E1.

36. Wu X, et al. Induction of neutralizing antibodies by human papillomavirus vaccine generated in mammalian cells. Antib Ther. 2019;2(2):45-53.

37. Li Z, et al. Increased prevalence of severe fever with thrombocytopenia syndrome in eastern China clustered with multiple genotypes and reasserted virus during 2010-2015. Sci Rep. 2017;7(1):6503.

38. Baral TN, MacKenzie R, Arbabi Ghahroudi M. Single-domain antibodies and their utility. Curr Protoc Immunol. 2013;103:2.17.1-2.17.57.

39. Jähnichen S, et al. CXCR4 nanobodies (VHH-based single variable domains) potently inhibit chemotaxis and HIV-1 replication and mobilize stem cells. Proc Natl Acad Sci U S A. 2010;107(47):20565-20570.

40. Yu Y, et al. Humanized CD7 nanobody-based immunotoxins exhibit promising anti-T-cell acute lymphoblastic leukemia potential. Int J Nanomedicine. 2017;12:1969-1983.

41. London NR, et al. Targeting Robo4-dependent Slit signaling to survive the cytokine storm in sepsis and influenza. Sci Transl Med. 2010;2(23):23ra19. 